\title{
Relevance of spectral cues for auditory spatial processing in the occipital cortex of the blind
}

\author{
Patrice Voss ${ }^{1,2}$, Franco Lepore ${ }^{3}$, Frédéric Gougoux ${ }^{3}$ and Robert J. Zatorre ${ }^{1,2 *}$ \\ ' Montreal Neurological Institute, McGill University, Montreal, QC, Canada \\ 2 International laboratory for Brain, Music and Sound Research, Montreal, QC, Canada \\ ${ }^{3}$ Centre de Recherche en Neuropsychologie et Cognition, Université de Montréal, Montreal, QC, Canada
}

Edited by:

Micah M. Murray, Université de

Lausanne, Switzerland

Reviewed by:

Alexander Stevens, Oregon Health and

Science University, USA

Melissa Saenz, University of Lausanne,

Switzerland

${ }^{*}$ Correspondence:

Robert J. Zatorre, Department of

Neuropsychology, Room 256, 3801 rue

University, Montréal, QC, Canada H3A

$2 B 4$.

e-mail: robert.zatorre@mcgill.ca
We have previously shown that some blind individuals can localize sounds more accurately than their sighted counterparts when one ear is obstructed, and that this ability is strongly associated with occipital cortex activity. Given that spectral cues are important for monaurally localizing sounds when one ear is obstructed, and that blind individuals are more sensitive to small spectral differences, we hypothesized that enhanced use of spectral cues via occipital cortex mechanisms could explain the better performance of blind individuals in monaural localization. Using positronemission tomography (PET), we scanned blind and sighted persons as they discriminated between sounds originating from a single spatial position, but with different spectral profiles that simulated different spatial positions based on head-related transfer functions. We show here that a sub-group of early blind individuals showing superior monaural sound localization abilities performed significantly better than any other group on this spectral discrimination task. For all groups, performance was best for stimuli simulating peripheral positions, consistent with the notion that spectral cues are more helpful for discriminating peripheral sources. PET results showed that all blind groups showed cerebral blood flow increases in the occipital cortex; but this was also the case in the sighted group. A voxel-wise covariation analysis showed that more occipital recruitment was associated with better performance across all blind subjects but not the sighted. An inter-regional covariation analysis showed that the occipital activity in the blind covaried with that of several frontal and parietal regions known for their role in auditory spatial processing. Overall, these results support the notion that the superior ability of a sub-group of early-blind individuals to localize sounds is mediated by their superior ability to use spectral cues, and that this ability is subserved by cortical processing in the occipital cortex.

Keywords: blindness, auditory spatial processing, spectral cues, functional neuroimaging, PET, crossmodal reorganization visual cortex plasticity

\section{INTRODUCTION}

Blindness has provided the scientific community with an exceptional model of how the brain is able to adapt to significant alterations within its environment. An emerging consensus suggests that blind individuals, especially those deprived from birth, show massive reorganization of function in cortical areas normally dedicated to vision such that they become responsive to other sensory modalities (see Merabet and Pascual-Leone, 2010; Voss et al., 2010 for reviews). These reorganizations are often accompanied by behavioral enhancements, most notably in the auditory and tactile domains, although these are not widespread general enhancements but are rather revealed under particular conditions. One area vastly studied is that of auditory localization, which is highly relevant considering the strong ecological value of being able to localize sound sources for proper and safe navigation within one's environment when visual cues are no longer available.

Despite the initial view that blind individuals might be at a disadvantage, given the important role vision has in shaping auditory space (Axelrod, 1959; Jones, 1975), most subsequent studies have shown that blind individuals are, at the very least, as good as sighted subjects when it comes to localizing sounds (see Collignon et al.,
2009 for a review). In fact, a sub-sample of early blind individuals has been shown to possess superior localization abilities when tested under particular conditions such as monaural sound localization (Lessard et al., 1998; Gougoux et al., 2005). Several studies have also investigated the neural substrate of auditory spatial processing in the blind and have shown that it elicits the participation of visual cortices (Weeks et al., 2000; Gougoux et al., 2005; Voss et al., 2008). The Gougoux et al. (2005) study was particularly instrumental in first demonstrating the functional role of the visual cortex for sound localization by showing significant correlations between performance in a monaural sound localization task and cerebral blood flow (CBF) in several occipital regions. More recent fMRI findings strongly suggest a functional specialization of the right dorsal visual cortex for auditory spatial processing in the blind (Collignon et al., 2010; Renier et al., 2010).

Still unanswered is why the observed enhancements are specific to conditions like monaural sound localization. An obvious explanation would be that blind individuals make better use of the monaural cues available to them. One such cue, resulting from the spectral filtering of the incoming sound wave by the outer ear (Middlebrooks and Green, 1991), referred to as spectral cues, has 
often been proposed as being more efficiently processed by blind individuals. To address this hypothesis, Doucet et al. (2005) showed that the supranormal performance of early blind individuals in a monaural localization task was decreased by the occlusion of the pinna or by the high- and low-pass filtering of the stimuli, suggesting that use of spectral cues is important for proper localization. However others have argued that other monaural cues could be responsible to for the superior abilities of the blind. For instance, Lewald (2002) proposed instead that blind individuals better process audiomotor feedback and proprioceptive cues relating to head position, whereas Van Wanrooij and Van Opstal (2004) have elegantly illustrated the importance of head-shadow cues for monaural localization. In addition, both Morgan (1999) and Zwiers et al. (2001) have argued that the fixed intensity of the sound target allows for the use of perceived sound intensity as an alternative localization cue.

Therefore, in an attempt to resolve this disputed issue, we designed a study aimed at examining the importance of spectral cues in the processing of spatial information in blind subjects using a novel approach, by presenting stimuli from which all other cues would be removed, leaving only the spectral content to be processed. More specifically, we asked blind and sighted controls to discriminate between sounds that were presented from a single spatial position, but that had different spectral profiles which simulated different spatial positions based on head-related transfer functions (HRTF) measurements. Our goal was not only to evaluate the behavioral aspects of spectral perception, but of particular interest to us was establishing the neural substrate associated with HRTF processing in the blind, and determining whether or not it is similar to that observed for monaural free-field sound localization. This is a critical question because the previous studies showing occipital recruitment in monaural localization (Gougoux et al., 2005) have not demonstrated that similar recruitment would occur to non-spatial spectral processing; this would be necessary in order to be able to conclude that spectral processing is responsible both for the improved behavioral performance and the activation in occipital regions. To address this issue, we used positron-emission tomography (PET) to investigate which cerebral regions are active while performing the non-spatial spectral task. We hypothesized that if blind subjects do make better use of spectral cues for localization, they should not only outperform the sighted subjects in a purely spectral discrimination task, in similar fashion to what is observed for monaural sound localization, but also show more task-driven responses in occipital areas. Furthermore, we separately studied early and late-onset blind individuals to study the effect that the age of onset of blindness would have on these measures. We hypothesized that an earlier onset should lead to both better performances and increased occipital recruitment, as was the case for monaural sound localization.

\section{MATERIALS AND METHODS PARTICIPANTS}

The participants were 7 healthy sighted volunteers (four males; three females) who ranged in age from 22 to 48 years (mean of 27.9 years), 6 late-onset blind (LB) individuals (three males; three females) who ranged in age from 33 to 54 years (mean of 41.5 years), and 12 early-onset blind (EB) individuals (six males; six females) who ranged in age from 21 to 41 years (mean of 31.3 years). In each case, the visual deficit resulted from anomalies in peripheral structures and led to total blindness except for some residual light perception in two LB subjects and in three EB subjects (categories 4 and 5, according to the World Health Organization, 1989 classification). During testing, all subjects were instructed to keep their eyes shut to avoid any impact of light perception on the results. Onset of blindness ranged from 18 to 37 years (mean of 26.2 years) while duration of blindness ranged from 4 to 36 years (mean of 15.8 years) in the LB subjects. Onset of blindness ranged from 0 to 14 years (mean of 2.6 years) while duration of blindness ranged from 16 to 40 years (mean of 28.7 years) in the EB subjects. Audiometric thresholds were assessed for all participants and indicated normal and comparable hearing in both ears. All subjects gave written informed consent in accordance with the guidelines approved by the Montreal Neurological Institute and the Nazareth and LouisBraille Institute (NLBI) for the blind. The research protocols were approved by the ethics committees of the Centre de Recherche Interdisciplinaire en Réadaptation, which coordinates research with blind subjects sponsored by the NLBI, the Montreal Neurological Institute, where the PET scans were carried out, and the Université de Montréal, from which the project originated.

All blind and sighted subjects had previously participated in auditory spatial studies in our lab (early: Gougoux et al., 2005; Voss et al., 2008; late: Voss et al., 2006, 2008). The EB subjects were further divided into two sub-groups based on their precision during a monaural sound localization task performed inside an anechoic chamber (see Gougoux et al., 2005). Those who could localize the sounds more accurately than sighted control subjects formed the "early-blind with superior performance" (EBSP) group, the remaining group consisted of those who showed similar performance to that of the sighted ("early-blind with normal performance"; EBNP). A similar separation was not possible with the LB because none of them had shown superior performance in the sound localization tasks.

\section{TASK AND PROCEDURE}

The stimuli were a pair of $30 \mathrm{~ms}$ noise bursts filtered with HRTF measurements (see Figure 1 for comparison of stimuli spectrograms) recorded from one ear of a KEMAR mannequin ${ }^{1}$ (see Gardner and Martin, 1994 for a full description of how the HRTF measurements were performed). The stimuli were always delivered in the scanner through a single loudspeaker located directly in front of the subject's head (this loudspeaker was part of a semicircular array of speakers that could be inserted into the PET scanner and is described elsewhere in further detail (Gougoux et al., 2005)). Thus, although the stimuli were derived from a spatially relevant filter, the result was pairs of noise bursts that differed only in terms of their spectral energy distribution (Figure 1), and hence perceptually, their timbre. The stimuli were presented at $60 \mathrm{~dB}$ SPL, with an inter-stimulus interval of $500 \mathrm{~ms}$ and an inter-trial interval of $1500 \mathrm{~ms}$. Each stimulus simulated one of the following spatial positions along the azimuthal plane: $\pm 60^{\circ}, \pm 45^{\circ}, \pm 30^{\circ}, \pm 15^{\circ}$, and $0^{\circ}$; the second stimulus either simulated the same position or

${ }^{1}$ http://sound.media.mit.edu/resources/KEMAR.html 


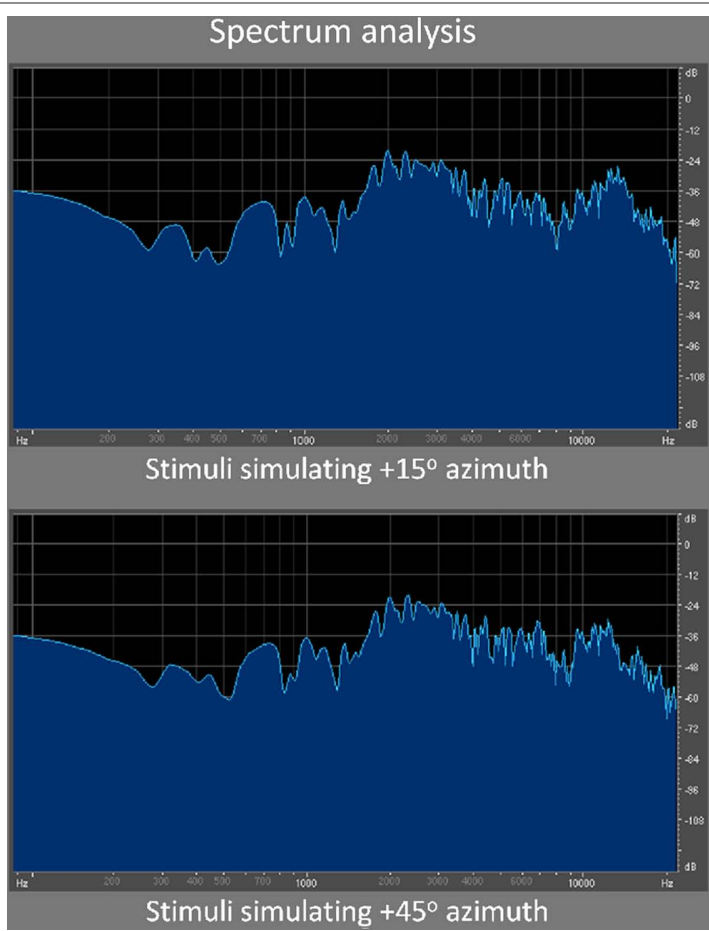

FIGURE 1 | Spectrum analysis. Here are illustrated the spectrograms of two sample stimuli used in the discrimination task. Specifically, these two stimuli were often compared to one another when the reference (first) stimulus was simulating $+15^{\circ}$ azimuth. As can be seen, the differences in spectral content between both sounds are very subtle, consistent with the difficulty level of the task.

another position $\pm 30^{\circ}$ from the first stimulus. Subjects clicked on a two-button keypad to indicate the response ("same" or "different"). A total of 112 trials were presented to each subject following a pseudo-random presentation, with an equal number of "same" and "different" trials. The behavioral tasks were presented around $15 \mathrm{~s}$ prior to the onset of data acquisition with the scanner. A baseline condition was acquired that consisted in alternate clicking of the left and right buttons after hearing a stimulus pair of identical unfiltered noise bursts presented in the frontal position. The subjects were informed prior to this baseline condition that all the sounds would always emulate a single central spatial position. All subjects were instructed to keep their eyes closed during the tasks and scanning procedures.

\section{IMAGING PROTOCOL AND ANALYSIS}

Cerebral blood flow was measured with a Siemens HR+ scanner and the $\mathrm{H}_{2} \mathrm{O}^{15}$ bolus method (Raichle et al., 1983). A T1-weighted MR image was also acquired to provide anatomical detail $(\mathrm{TE}=10 \mathrm{~ms}$; $\mathrm{TR}=22 \mathrm{~ms}$, sagittal acquisition plane, head coil, flip angle $30^{\circ}$ ) on a 1.5-T Siemens Magnetom scanner. CBF images were passed through a 14-mm Hanning filter, normalized for differences in global CBF, and co-registered with the individual MRI data (Evans et al., 1992). Each MRI/PET dataset was then linearly resampled into a standardized stereotaxic coordinate system based on the MNI305 target (a sample of 305 normal subjects) via an automated feature-matching algorithm (Collins et al., 1994), resulting in a normalized brain space similar to the Talairach and Tournoux (1988) atlas. PET images were averaged across subjects for each condition, and the mean change image volume was obtained for each comparison; this volume was converted to a $t$-statistic map, and the significance of focal CBF changes was assessed by a method based on three-dimensional Gaussian random-field theory (Worsley et al., 1992). The presence of significant changes in CBF was first established on the basis of an exploratory search, for which the $t$-value criterion was set at 3.53 or greater. This value corresponds to an uncorrected $p$-value of 0.0004 (two-tailed), and results in an average of 0.58 false positives per search volume of 182 resolution elements (each of which has dimensions of $14 \mathrm{~mm} \times 14 \mathrm{~mm} \times 14 \mathrm{~mm}$ ), corresponding approximately to the volume of gray matter scanned. Occipital activations were considered when above $t=3.00$ given our a priori assumptions regarding these areas based on our previous findings. Covariation analyses between CBF changes and non-imaging measures followed the procedure outlined by Paus et al. (1996).

\section{INTER-REGIONAL CORRELATION ANALYSIS}

We also investigated the functional connectivity of specific seed areas in the occipital cortex of the blind subjects. For the discrimination condition, normalized $\mathrm{rCBF}$ rates were correlated, across different sub-groups of blind subjects, with values derived from seed voxels of interest established a posteriori based on activation peaks and areas whose activity significantly correlated with performance. This was achieved by utilizing software written as part of the SurfStat package ${ }^{2}$, generating a normalized output image with a correlation coefficient assigned to each voxel, indexing correlations between blood flow in that voxel and the seed voxel of interest. The strength of the inter-regional relationship was assessed by an across-subject correlation, a high correlation coefficient indicating that a region is likely to be functionally connected with the reference region. The correlation maps were then converted to a $t$-statistic map and threshold for significance was set at $t=4.18$, corresponding to an uncorrected $p$-value of 0.004 (two-tailed).

\section{RESULTS}

\section{BEHAVIORAL RESULTS}

As described in the "Materials and Methods" Section, the early blind group was divided into two sub-groups based on their performances during a previously performed monaural localization task (Gougoux et al., 2005): one showing normal performance as compared to the sighted subjects (EBNP; seven subjects) and one showing superior performance (EBSP; five subjects).

The behavioral results from the current spectral discrimination task are plotted in Figure 2. As can be seen, one group (EBSP) clearly separates itself from the rest by showing superior overall performance in the discrimination task [repeated measures ANOVA: $\left.F_{(3,21)}=7.548 ; p \leq 0.001\right]$. Post hoc Tukey tests confirmed that only the EBSP group's performance significantly differed from the rest (vs. EBSP, $p=0.005$; vs. LB, $p \leq 0.001$; vs. SIG, $p=0.03$ ). All other comparisons were not significant. Finally, performance was better

${ }^{2}$ http://www.math.mcgill.ca/keith/surfstat 


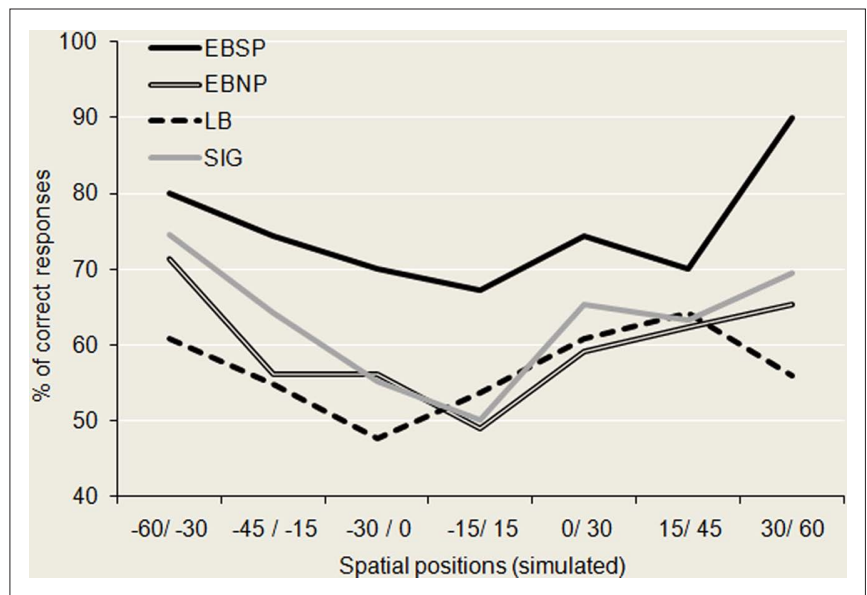

FIGURE 2 | Behavioral performance for the spectral discrimination task. Here are plotted the performance of each group across the different azimuthal regions tested. The EBSP group significantly outperformed all other groups while all other comparisons were non-significant. Noteworthy is the finding that performance is best for spectral cues corresponding to peripheral positions, for all groups, consistent with the notion that spectral cues are most beneficial for peripheral auditory space in the horizontal plane.

for auditory stimuli simulating peripheral positions for all groups as evidenced by a 2 (position: center/periphery) $\times 4$ (group) ANOVA where the results for the three most central positions were averaged into a "center" variable and those for the four most lateral were averaged into a "periphery" variable $\left[F_{(1,21)}=14.893 ; p \leq 0.001\right]$. There was no group $\times$ position interaction, suggesting that all groups were equally better for peripheral positions compared to central ones.

\section{IMAGING RESULTS}

Compared to the control condition, all groups showed CBF increases in the occipital cortex (see Figure 3) with significant peaks in right Cuneus (EBSP), right lingual gyrus (EBNP, LB), left lingual gyrus (LB), and left inferior occipital gyrus (LB) when performing the spectral discrimination task (see Table 1 for complete list of significant foci). Unexpectedly, the sighted group also showed occipital peaks in some areas. In contrast, significant occipital deactivations were also observed in both the sighted group and the EBNP group in similar areas of the right middle occipital gyrus. Direct intergroup contrasts showed that all blind groups had significantly increased activity in the occipital cortex compared to sighted controls, most notably in the right cuneus (see Figure 4, top row). However, the sighted group also had significantly increased activity in posterior occipital areas relative to the other blind groups (see Figure 4, bottom row), most notably in the lingual gyrus. The complete list of significant differences between groups is listed in Table 2A.

\section{CORRELATIONAL ANALYSES}

Independent voxel-wise covariation analyses were carried out across the entire group of blind individuals to test whether brain activity could be directly linked to behavioral and subject measures. Two such analyses were performed across all blind subjects, irrespective of the group to which they had been assigned. First, the individual overall corrected response percentage score, and second, the age of onset of blindness for each subject, were entered as regressors in two separate analyses examining covariation with CBF change across the entire brain volume, following the procedure outlined by Paus et al. (1996). Performance was found to correlate with activity in the left lingual gyrus $(r=0.56, p=0.015)$, and also with activity near the left precentral sulcus/inferior frontal cortex boundary ( $r=0.46, p=0.05$; see Figure 5). However, contrary to previous findings with the same subject sample (Gougoux et al., 2005; Voss et al., 2008), no occipital area activity was found to vary as a function of the age of onset of blindness. Moreover, the age of onset of blindness also failed to predict performance ( $r=-0.28, p=0.25$ ), again contrary to previous findings (Voss et al., 2008).

\section{INTER-REGIONAL CORRELATION ANALYSES}

Considering that the exact manner in which the occipital cortex is recruited by non-visual inputs/tasks remains elusive, we examined the task-defined occipital seed regions in inter-regional correlation analyses to determine what regions could be functionally connected to them. Given the small number of subjects in each blind group, however, within subgroup inter-regional correlations did not approach significant levels. We then proceeded to an analysis including all blind subjects and using as seed point the area whose activity was shown to positively correlate with performance [left lingual gyrus (tal. coord.: $-8,-102,-5$ ); see Figure 5]. The activity in four regions was found to significantly covary with the activity of the seed region across all blind subjects (see Figure 6; Table 2B): right inferior frontal gyrus, right middle frontal gyrus, left middle frontal gyrus, and right superior parietal lobule.

\section{DISCUSSION}

This study was designed to assess the role of spectral cue processing in blind individuals and its neural substrate. We show here that the performance of blind individuals, when discriminating noise bursts using spectral cues that mimic those relevant for spatial localization, follows the same pattern as when these same individuals localize or discriminate between actual sound sources monaurally (Gougoux et al., 2005; Voss et al., 2008). In each case, only the EBSP group was able to outperform all groups. Similarly, a significant positive correlation between performance in the spectral discrimination task and posterior activation peaks in the occipital cortex of blind individuals was established, as had been shown for the aforementioned spatial tasks (Gougoux et al., 2005; Voss et al., 2008), adding further support to the notion that occipital areas in the blind appear to play a functional role in auditory spatial processing. This conclusion is perhaps best supported by the study of Collignon et al. (2007), which showed that the sound localization performance of blind subjects was significantly poorer following the transient disruption of their occipital cortex by repetitive transcranial magnetic stimulation. Finally, the age of onset of blindness did not predict behavioral performance in the discrimination task nor did it predict occipital activation patterns in the blind individuals. This is in contrast with the data obtained with a sound source discrimination task (Voss et al., 2008) performed by the same subject sample, where the age of onset of blindness was found to predict both of these measures. This is essentially due to the fact that in the current study both 
Table 1 | Stereotaxic coordinates and $t$-values of activation and deactivation foci (coordinates refer to standardized stereotaxic space (Talairach and Tournoux, 1988)).

\begin{tabular}{|c|c|c|c|c|c|c|c|c|}
\hline & \multicolumn{4}{|c|}{ Areas (increases) } & \multicolumn{4}{|c|}{ Areas (decreases) } \\
\hline & $x$ & $y$ & $z$ & $t$-score & $x$ & $y$ & $z$ & $t$-score \\
\hline \multicolumn{9}{|l|}{ EBSP } \\
\hline R inferior frontal gyrus & 39 & 49 & 0 & 5.38 & & & & \\
\hline R middle frontal gyrus & 40 & 32 & 24 & 4.57 & & & & \\
\hline $\mathrm{L}$ inferior frontal gyrus & -17 & 46 & -18 & 4.13 & & & & \\
\hline $\mathrm{R}$ inferior frontal gyrus & 20 & 44 & -15 & 3.92 & & & & \\
\hline L medial frontal gyrus & -1 & 18 & 51 & 3.90 & & & & \\
\hline$R$ cuneus & 12 & -81 & 9 & 3.80 & & & & \\
\hline R inferior frontal gyrus & 54 & 15 & 14 & 3.75 & & & & \\
\hline $\mathrm{R}$ angular gyrus & 50 & -49 & 50 & 3.75 & & & & \\
\hline L/R precuneus & & & & & 0 & -57 & 21 & -3.78 \\
\hline L medial frontal gyrus & & & & & -4 & -18 & 54 & -3.71 \\
\hline R cingulate cortex & & & & & 3 & 41 & -11 & -3.60 \\
\hline L medial frontal gyrus & & & & & -7 & -26 & 59 & -3.60 \\
\hline R precentral gyrus & & & & & 23 & -23 & 54 & -3.56 \\
\hline \multicolumn{9}{|l|}{ EBNP } \\
\hline $\mathrm{R}$ inferior frontal gyrus & 44 & 42 & 0 & 4.38 & & & & \\
\hline $\mathrm{R}$ lingual gyrus & 11 & -83 & -11 & 4.32 & & & & \\
\hline R inferior frontal gyrus & 36 & 22 & 5 & 3.77 & & & & \\
\hline$L$ inferior frontal gyrus & -39 & 49 & -3 & 3.68 & & & & \\
\hline $\mathrm{R}$ angular gyrus & 48 & -48 & 51 & 5.38 & & & & \\
\hline R cerebellum & 24 & -76 & -21 & 5.15 & & & & \\
\hline$L$ inferior frontal gyrus & -23 & 36 & -21 & 4.84 & & & & \\
\hline $\mathrm{R}$ middle frontal gyrus & 39 & 48 & 17 & 4.70 & & & & \\
\hline R inferior frontal gyrus & 20 & 42 & -17 & 4.70 & & & & \\
\hline R middle frontal gyrus & 39 & 32 & 27 & 4.60 & & & & \\
\hline R inferior frontal gyrus & 38 & 22 & 6 & 4.56 & & & & \\
\hline R medial frontal gyrus & 3 & 29 & 39 & 4.41 & & & & \\
\hline R middle frontal gyrus & 47 & 37 & 18 & 4.37 & & & & \\
\hline L medial frontal gyrus & -1 & 18 & 53 & 4.30 & & & & \\
\hline L inferior frontal gyrus & -36 & 53 & 3 & 4.15 & & & & \\
\hline R middle frontal gyrus & 52 & 20 & 32 & 4.02 & & & & \\
\hline $\mathrm{R}$ lingual gyrus & 15 & -85 & -20 & 3.90 & & & & \\
\hline$R$ lingual gyrus & 21 & -92 & -11 & 3.88 & & & & \\
\hline L brain stem & -8 & -28 & -3 & 3.88 & & & & \\
\hline R middle frontal gyrus & 38 & 15 & -12 & 3.73 & & & & \\
\hline L insula & -34 & 18 & 42 & 3.69 & & & & \\
\hline
\end{tabular}




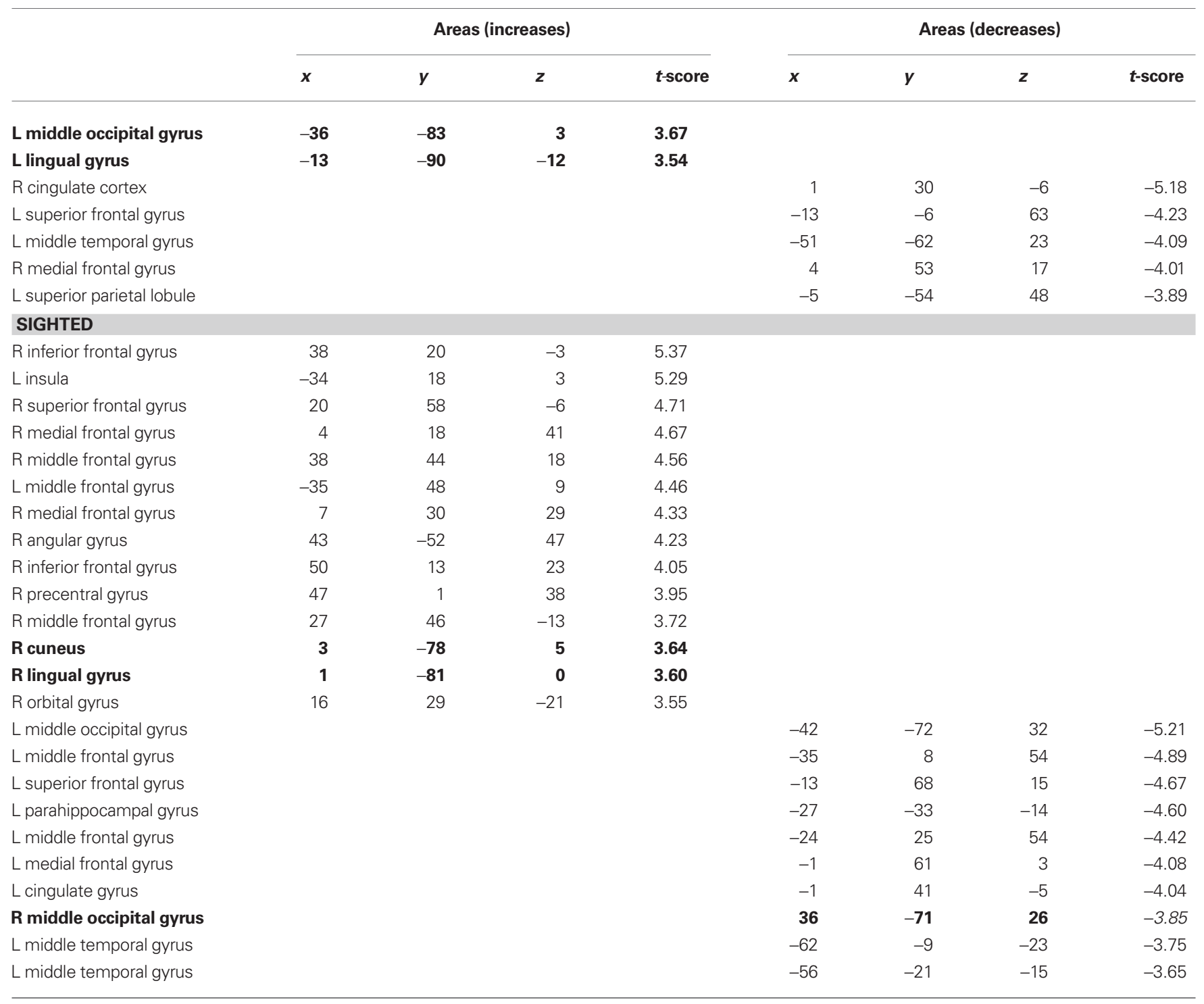

the EBNP and LB groups performed equally well, whereas in the previous study the EBNP preformed better than the LB, although that difference did not reach statistically significant levels.

\section{PROCESSING OF AUDITORY SPATIAL TARGETS BY THE BLIND}

Behavioral differences between blind and sighted individuals in auditory spatial tasks tend to manifest themselves under conditions that maximize the contribution of spectral cues, such as during monaural sound localization (Lessard et al., 1998; Gougoux et al., 2005) or during auditory spatial processing in peripheral auditory space (Röder et al., 1999; Voss et al., 2004; Després et al., 2005). Here we replicated previous behavioral findings using auditory targets with stimuli that were non-spatial in nature, but rather simulated spatial positions by using HRTF recordings from a KEMAR mannequin that were presented from a single central loudspeaker. As such, there was no spatial information present per se in the stimuli, only differences in spectral content, as all the sounds came from a single central loudspeaker (see Figure 1), and hence the discrimination was based on perceived differences in timbre. However, the spectral differences between the different stimuli approximately correspond to the difference in spectral changes that would have been created by the pinna had the stimuli been identical in spectral content but presented from different spatial positions. This conclusion is supported by our finding that performance is best for stimuli simulating more peripheral positions (see Figure 2), which is consistent with the notion that spectral cues gain importance as a function of eccentricity. These results therefore strongly support the hypothesis that early blind individuals' superiority in spatial auditory tasks rests on their better use of spectral cues to localize sounds in space (Lessard et al., 1998; Gougoux et al., 2005). Additional support for this hypothesis comes from the finding that blind individuals are more sensitive to small spectral differences in tone patterns than are sighted individuals (Gougoux et al., 2004; Wan et al., 2010). 


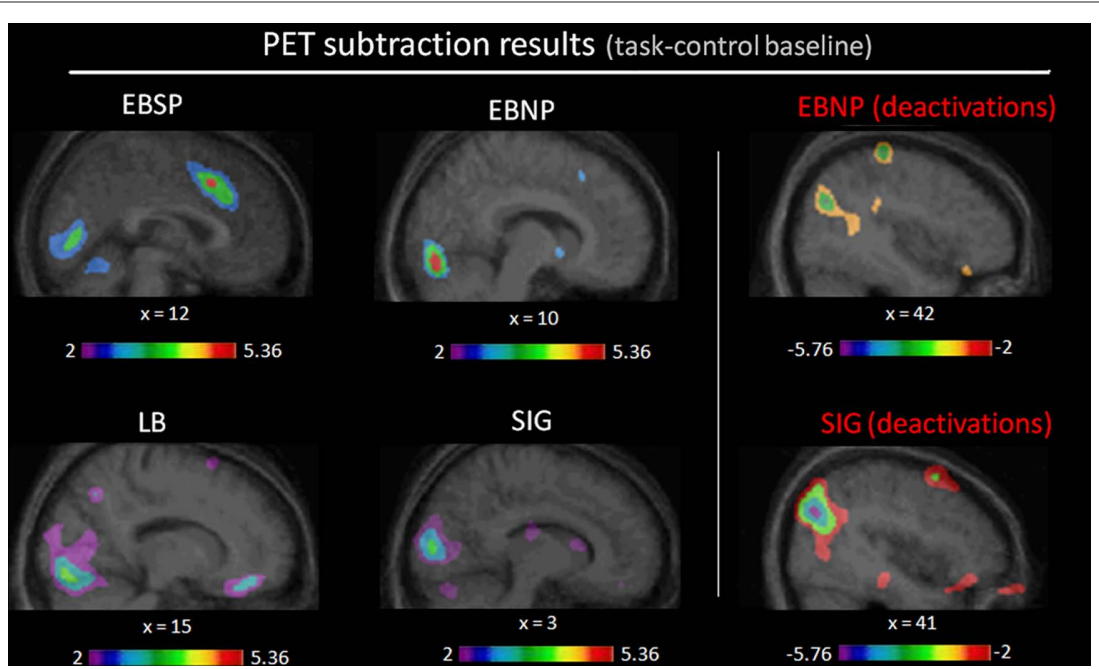

FIGURE 3 | Task-related contrasts for each group. Illustrated here are the occipital (de)activation foci generated by the task-related contrasts (task-control baseline) for each group (see Table 1 for complete list of significantly activated brain regions).

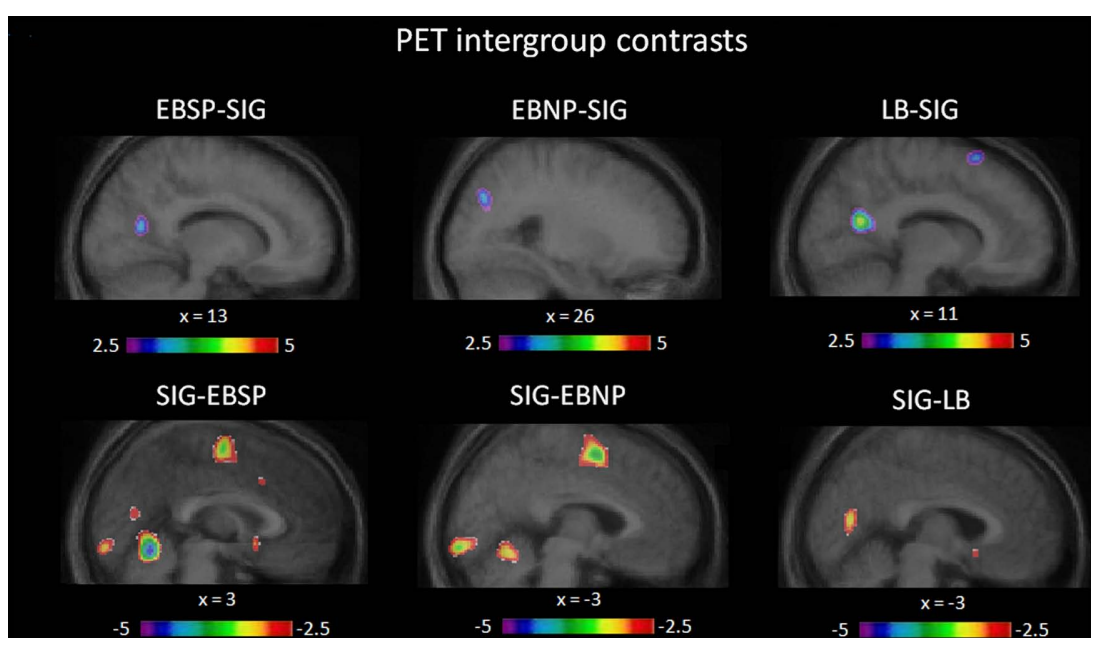

FIGURE 4 | Intergroup contrasts. Illustrated here are the differences in occipital activation between groups, relative to baseline, obtained via intergroup contrast analyses (see Table 2 for complete list of significant differentially activated brain regions).

What remains elusive is why the superior performance is only observed in a sub-sample of early blind individuals, and why enhanced performance in late-blind individuals has been observed when processing peripheral auditory targets (Voss et al., 2004; Fieger et al., 2006), but not during monaural auditory spatial tasks (Voss et al., 2006, 2008). The findings of Fieger et al. (2006) do shed some light on the issue. Using the same task that was used to show that early blind individuals outperformed sighted ones in an auditory spatial discrimination task (Röder et al., 1999), they showed that late-blind individuals also were better than their sighted counterparts. However, electrophysiological recordings showed that they did not differ from sighted individuals in their early perceptual response (N1 ERP component) as did the early blind individuals, but rather in their later attentional processing mechanisms (P3 ERP component), suggesting that compensatory mechanisms in adult onset blindness may differ from those seen following early onset blindness. Unfortunately, no such data are available to distinguish our EBSP and EBNP blind groups, making it difficult to account for the behavioral and brain activation differences. Both groups are nearly identical in age and in age of onset of blindness, while closer examination of the causes of blindness (see Gougoux et al., 2005 for detailed subject descriptions) gives little insight into what could be driving these differences. One possibility that should be further explored is whether or not the enhancements observed in the EBSP group are the result of experience-dependent adaptations. Perhaps these individuals learned at a very young age to pay 
Table 2 | Stereotaxic coordinates and $\boldsymbol{t}$-values of intergroup contrasts and functional connectivity analysis (coordinates refer to standardized stereotaxic space (Talairach and Tournoux, 1988)).

(A) Intergroup contrasts

\begin{tabular}{ccccccccc}
\multicolumn{3}{c}{ Areas (increases) } & \multicolumn{4}{c}{ Areas (decreases) } \\
\hline$x$ & $y$ & $z$ & t-score & $x$ & $y$ & $z$ & $t$-score
\end{tabular}

\section{EBSP MINUS EBNP}

L cerebellum

$L$ angular gyrus

$\begin{array}{rrrr}-28 & -50 & -21 & 3.93 \\ -58 & -47 & 45 & 3.77\end{array}$

$R$ post-central gyrus

$R$ superior frontal gyrus

$R$ cerebellum

\section{EBSP MINUS LB}

$R$ cerebellum

EBSP MINUS SIG

\section{$R$ cuneus}

13

$-64$

15

$R$ cerebellum

L postcentral gyrus

$R$ medial frontal gyrus

$L$ medial frontal gyrus

$R$ lingual gyrus

\section{EBNP MINUS SIG}

\section{$\mathrm{R}$ precuneus}

$L$ precuneus

$L$ middle temporal gyrus

$R$ cuneus/precuenus

L middle occipital gyrus

L/R Medial frontal gyrus

$R$ superior frontal gryus

$R$ medial frontal gyrus

$R$ middle frontal gyrus

\section{$L$ lingual gyrus}

\section{LB MINUS SIG}

$L$ middle frontal gyrus

$R$ cerebellum

\section{$R$ cuneus}

R precuneus

L inferior frontal gyrus

$R$ inferior frontal gyrus

$R$ inferior frontal gyrus

L putamen

$R$ lingual gyrus

\section{EBNP MINUS LB}

\section{$L$ inferior frontal gyrus}

R putamen

$R$ angular gyrus

$R$ middle frontal gyrus

$R$ cerebellum

$13 \quad-64 \quad 15 \quad 3.25 *$

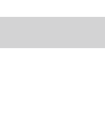

(2)

$\begin{array}{rrrr}40 & -28 & 57 & -4.97 \\ 11 & -2 & 58 & -3.62 \\ 5 & -59 & -11 & -3.56\end{array}$

$\begin{array}{llll}5 & -62 & -11 & -4.81\end{array}$




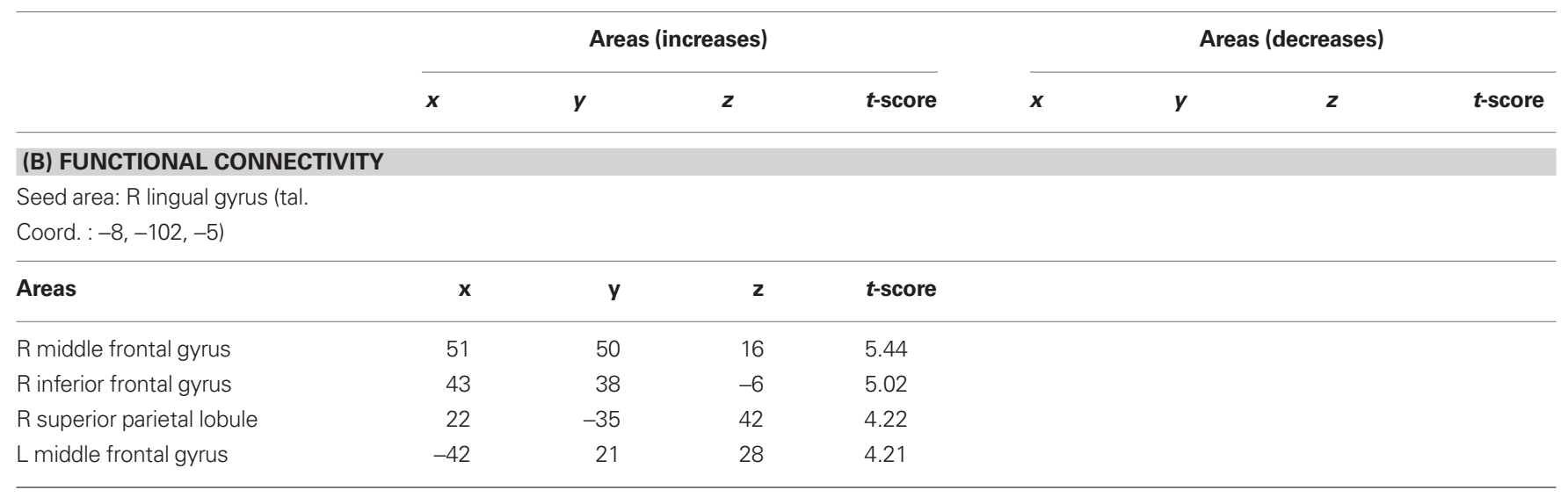

*Sub-threshold values.

attention to subtle spectral cues, whereas others did not, leading to experience-driven plasticity. Alternatively, the group disparity could also result from differential availability of neural resources. While occipital resources in some blind individuals might be dedicated to specific auditory functions, they might be dedicated to different functions or even to different modalities in others. Future studies might better disentangle the relative role of visually deafferented areas in auditory and tactile processing within the same subjects to ascertain if any differences could explain the observed dichotomy in auditory spatial processing in early blind individuals.

\section{OCCIPITAL ACTIVATION PATTERNS AND ROLE OF OCCIPITAL CORTEX IN THE BLIND}

We did find, as predicted, recruitment of occipital areas in the blind individuals when performing the spectral discrimination task, and more importantly, showed that the occipital cortex is functionally engaged via covariation analyses between $\mathrm{CBF}$ and performance measures. It is however somewhat difficult to explain the differences, and lack thereof, in the brain activation patterns between the blind groups. All three groups recruited occipital regions to carry out the discrimination task. Significant peaks were mostly located in the right hemisphere, with the exception of the LB who showed bilateral occipital recruitment. While the EBNP and LB groups recruited non-primary visual areas, the EBSP group recruited striate cortex (V1). Although the occipital region that appears to be most important for achieving good performance is also in V1, as shown via the covariation analyses, it is however situated in the left hemisphere. Notably, the EBNP group was the only one to also show decreased activity in the right middle occipital gyrus. However, despite all these differences, none of the groups differed significantly from one another in terms of occipital recruitment (or deactivation) during the task. It therefore appears that should these groups process the stimuli differently, they do so in a more subtle manner than these subtraction analyses are sensitive to. Moreover, the data indicate that activation patterns and intergroup contrasts do not tell the whole story, as evidenced by the whole-brain covariation analysis with performance, revealing that the activity in a different occipital area than those reported in the contrast analyses best predicted performance. This is consistent with our previous findings with the same subjects, where the occipital areas that best predicted performance were different from those that showed the largest increases elicited by auditory spatial tasks relative to baseline (Gougoux et al., 2005; Voss et al., 2008).

Interestingly, the occipital regions that best predict performance in the same blind subjects are different for each task: here (left lingual gyrus), monaural sound localization (right superior occipital gyrus, right ligual gyrus; Gougoux et al., 2005) and monaural sound source discrimination (left cuneus; Voss et al., 2008). While all tasks are slightly different from each other, they all share one key element: the fact that accurate processing of spectral information is essential for achieving high performance. This raises the question of whether the previously reported regions found to predict performance were also involved in spectral cue processing, or if the difference in location simply reflects the fact that other factors were in play as well, such as whether explicit localization was required or not, and whether other spatial cues were present in addition to spectral ones. Future studies will be needed to properly disentangle these issues.

In an attempt to better understand how non-visual input ends up being processed in visual areas and the nature of the processing being performed in these areas, we carried out an inter-regional covariation analysis to see which regions' activity would covary with that of visual areas. We chose a voxel within the left lingual gyrus as the seed region of the analysis as it was this region's activity that was found to best predict performance. The activity of four other cortical regions was found to significantly covary with that of the seed region: right middle frontal gyrus, right inferior frontal gyrus, the right superior parietal lobule, and the left middle frontal gyrus. These dorsolateral prefrontal and parietal regions are known for their role in auditory spatial processing and attention (Zatorre et al., 1999; Lewald et al., 2008), suggesting that these occipital cortices are now part of an integrated auditory spatial network. This could be achieved through a fairly straightforward crossmodal transition since these frontal are parietal regions are also known for their general role in spatial processing and spatial attention in the visual modality (see Silver and Kastner, 2009; Szczepanski et al., 2010), and thus are important for spatial processing/attention regardless of the input modality. This finding is consistent with those of Weeks et al. (2000) who also showed inter-regional correlations in blind subjects between right occipital cortex and a network of regions important for auditory spatial processing. 


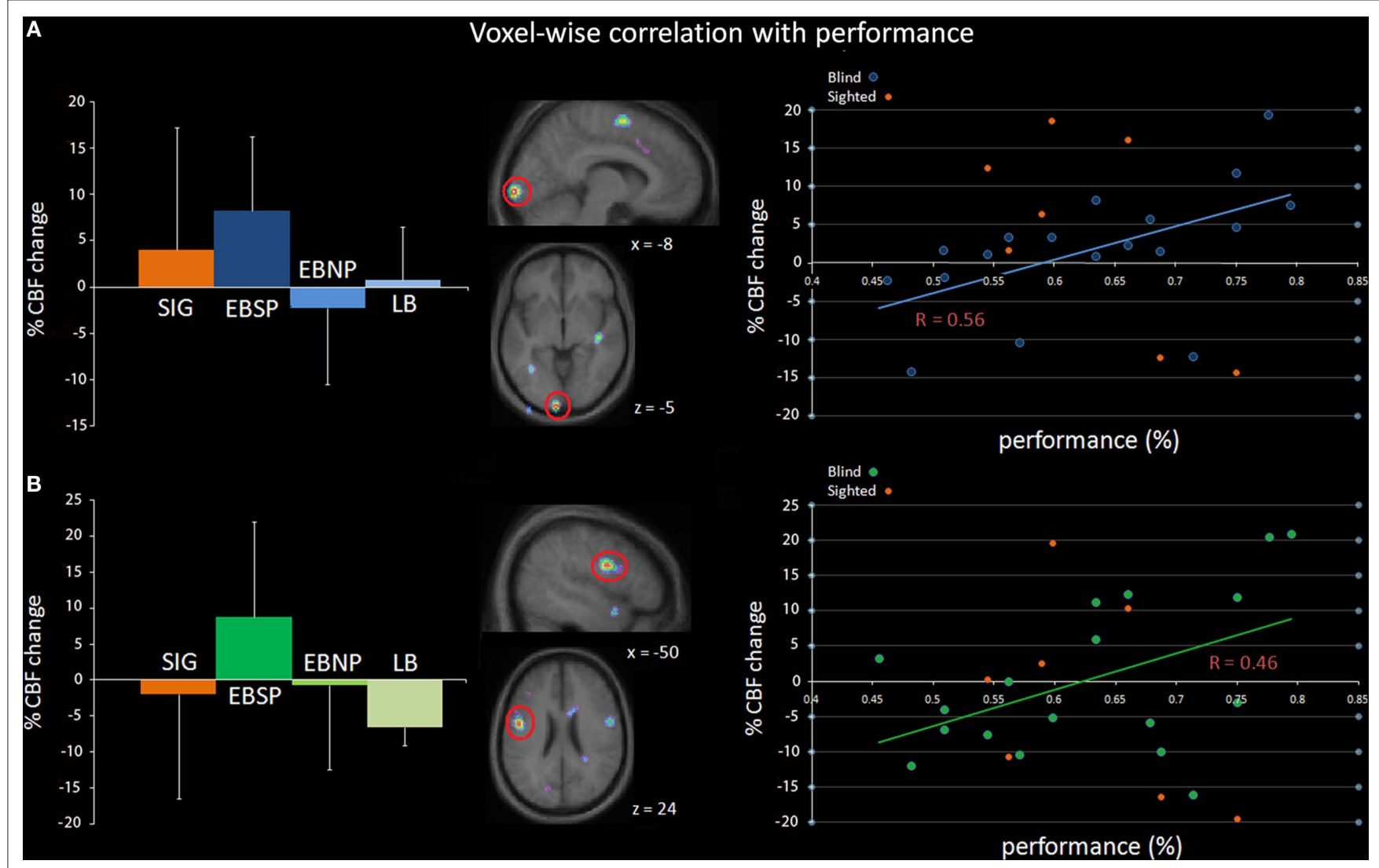

FIGURE 5 | Independent voxel-wise covariation analysis in the blind. The percentage of CBF change (relative to baseline) significantly correlated with performance of blind subjects scores in two regions: (A) left lingual gyrus (tal. coord.: $-8,-102,-5)$ and (B) the left precentral gyrus/inferior frontal gyrus (tal. coord.: $-50,-2,24)$. The left column illustrates the average percent CBF change per group for each region relative to baseline while the middle column shows sagittal and horizontal slices highlighting the two regions. The right column illustrates the correlation between percent CBF change and performance across all blind subjects. Note that the data points for sighted subjects are also included in the scatter plot for comparison purposes, but were not included in the correlation analysis.
Particularly interesting is the finding that regions known to be part of an auditory spatial network are also involved in and important for auditory spectral processing (Zatorre et al., 1999; Lewald et al., 2008), suggesting the existence of an intertwined relationship between the processing of spectral information and spatial information, thus reinforcing the notion that the analysis of spectral cues can be a determining factor in spatial processing. Indeed, if the occipital cortex of some early blind individuals has tapped into an auditory network involved both in spatial and spectral processing; it naturally follows that their performance in spatial tasks would improve as a result of enhanced processing of spectral cues.

\section{OCCIPITAL PROCESSING IN THE SIGHTED}

An unexpected finding is the occipital activation peak observed in the sighted group, especially considering that these same subjects participated in two previous studies where no occipital recruitment was observed during auditory spatial tasks (Gougoux et al., 2005; Voss et al., 2008). While there has been a recent accumulation of reports that short periods of visual deprivation can lead to such crossmodal changes (Pascual-Leone and Hamilton, 2001; Weisser et al., 2005; Merabet et al., 2008), the present findings show that auditory stimuli can activate primary visual areas in the absence of prolonged deprivation. Similar evidence was previously obtained by Poirier et al. (2005) when they showed that moving auditory targets can activate the MT/V5 region near the occipital/temporal junction. More recently, Zangenehpour and Zatorre (2010) showed that occipital regions in the sighted were responsive to auditory input following brief exposures to audiovisual stimuli, thus reinforcing the notion that auditory and visual cortices interact to a larger degree than previously thought. While in these prior studies the functional relevance of the activations was rather straightforward in interpretation, establishing the functional significance of the activation observed in the sighted here is not as clear-cut. As seen in the scatter plot Figure 5, there is no positive relationship between performance and occipital activation, which contrasts with the EBSP in whom activation was predictive of performance. We therefore tend to favor the hypothesis that the occipital recruitment in the sighted may not be related to task performance, and hence may be an epiphenomenon of some sort.

Also unclear is whether the occipital activation seen in the sighted are actually auditory-driven or not. Although the sighted subjects did not show any occipital activation in our previous auditory spatial 


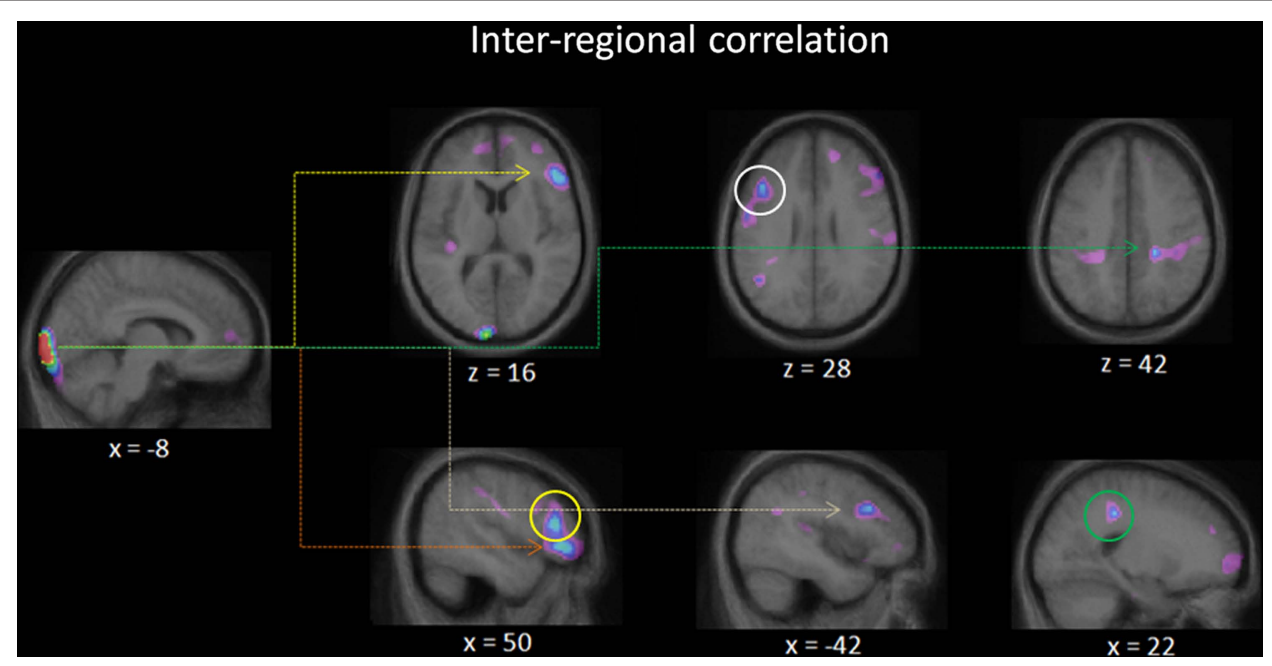

FIGURE 6 | Inter-regional correlation analysis. Illustrated here are the regions in which the activity covaried with that of the activity of a user defined seed region: the left lingual gyrus centered at tal. coord.: $(-8,-102,-5)$. The seed point corresponds to the voxel whose activity most correlated with performance across all blind individuals (see Figure 5). Four peaks reached above threshold values for significant covariation: right inferior frontal gyrus (orange arrow), right middle frontal gyrus (yellow arrow, circle), left middle frontal gyrus (white arrow, circle), and right superior parietal lobule (green arrow, circle). studies, one cannot rule out the possibility that they might have used mental visualization in the current experiment. Contrary to the previous studies, there was a complete absence of non-spectral spatial cues in the current task, which perhaps lead the subjects to attempt some form of visual imagery to aid them in performing this difficult task. Interestingly, one other study has shown the right occipital cortex to be implicated in pitch processing in sighted individuals (Zatorre et al., 1994). However, given the lack of a systematic observation of occipital recruitment in such tasks throughout the literature, it is difficult to speculate any further on the reasons for occipital recruitment in sighted individuals elicited by spectral discrimination tasks.

Perhaps more relevant is the observation of occipital deactivation in the sighted. This finding was expected based on previous findings with sighted subjects performing auditory tasks (Weeks et al., 2000; Gougoux et al., 2005; Voss et al., 2008), and is often interpreted as resulting from crossmodal inhibition in the unattended modalities (Kawashima et al., 1995; Shulman et al., 1997; Laurienti et al., 2002; Johnson and Zatorre, 2005). Should the occipital activation in the sighted actually be auditory-driven, it would constitute support for simultaneous activation and deactivation of different occipital areas following non-visual input. Using a tactile exploration task, Merabet et al. (2007) showed that V1 was strongly activated while secondary visual areas were deactivated. The authors proposed that tactile processing probably affects occipital cortex via two separate pathways: a suppressive top-down pathway descending through the visual cortical hierarchy and an excitatory pathway arising from outside the visual cortical hierarchy that drives area V1 directly. A similar pattern may be present in the current data.

\section{CONCLUSION}

We have shown here that the performance of blind individuals when discriminating noise bursts in a non-spatial context using spectral cues that mimic those relevant for spatial localization follows the same pattern as when these same individuals localize actual sound sources monaurally. In general, the brain activation patterns were also consistent with previous findings related to monaural sound localization, with the main exception of significant occipital foci observed in the sighted. It is unclear whether this finding relates to multimodal processing in the occipital cortex of the sighted or if visual imagery was involved. Overall, these results strongly support the notion that the superior ability of a sub-group of early blind individuals to localize sounds is mediated by their superior ability to use spectral cues, and that this ability is subserved by cortical processing in the occipital cortex. Moreover, we also show that the occipital activity in the region that best predicted performance also covaried with several frontal and parietal regions known for their importance in auditory spatial processing, thus supporting the interpretation that occipital areas in these blind individuals form part of a functional network for spatial analysis.

\section{ACKNOWLEDGMENTS}

We firstly thank the participants, as well as the Nazareth and Louis-Braille Institute (NLBI), the Montreal Association for the Blind, the Regroupement pour les Aveugles et Amblyopes de Montreal, Charles Leclerc, Marie-Eve Doucet, Nadia Lessard for their assistance in recruiting the blind subjects. We also thank Kate Hanratty, Pierre Ahad, Marc Bouffard, Francine Giroux, Sylvain Milot, Robert Lisbonna, Gary Sauchuck, Rick Fukasawa, Alan Evans, and Bruce Pike for technical and statistical assistance. We also thank Bill Gardner and Keith Martin from the MIT Media Lab for making the stimuli available to us. This study was supported by grants from the Canadian Institutes of Health Research (CIHR), the Natural Sciences and Engineering Research Council of Canada (NSERC), and by the Fonds de Recherche en Santé du Québec (FRSQ). 


\section{REFERENCES}

Axelrod, S. (1959). Effects of Early Blindness. New York: American Foundation for the Blind.

Collignon, O., Lassonde, M., Lepore, F., Bastien, D., and Veraart, C. (2007). Functional cerebral reorganization for auditory spatial processing and auditory substitution of vision in early blind subjects. Cereb. Cortex 17, 457-465.

Collignon, O., Vandewalle, G., Voss, P., Albouy, G., Charbonneau, G., Lassonde, M., and Lepore, F. (2010). Functional specialization for auditoryspatial processing reveals modularity in the reorganized "visual" cortex of congenitally blind humans. Proc. Natl. Acad. Sci. U.S.A.

Collignon, O., Voss, P., Lassonde, M., and Lepore, F. (2009). Cross-modal plasticity for the spatial processing of sounds in visually deprived subjects. Exp. Brain Res. 192, 343-358.

Collins, D. L., Neelin, P., Peters, T., and Evans, A. (1994). Automatic 3D intersubject registration of $\mathrm{MR}$ volumetric data in standardized Talaraich space. $J$. Comput. Assist. Tomogr. 18, 192-205.

Després, O., Candas, V., and Dufour, A. (2005). The extent of visual deficit and auditory spatial compensation: evidence from self-positioning from auditory cues. Cogn. Brain Res. 23, 444-447.

Doucet, M. E., Gagné, J. P., Leclerc, C., Lassonde, M., Guillemot, J. P., and Lepore, F. (2005). Blind subjects process auditory spectral cues more efficiently than sighted people. Exp. Brain Res. 160, 194-202.

Evans, A., Marrett, S., Neelin, P., Collins, L., Worsley, K., Dai, W., Milot, S., Meyer, E., and Bub, D. (1992). Anatomical mapping of functional activation in stereotaxic coordinate space. Neuroimage 1, 43-53.

Fieger, A., Röder, B., Teder-Sälejärvi, W., Hillyard, S. A., and Neville, H. J. (2006). Auditory spatial tuning in lateonset blindness in humans. J. Cogn. Neurosci. 18, 149-157.

Gardner, B., and Martin, K. (1994). HRTF Measurements of a KEMAR DummyHead Microphone MIT Media Lab PerceptualComputing-TechnicalReport \#280. Available at: http://sound.media. mit.edu/resources/KEMAR/hrtfdoc.txt

Gougoux, F., Lepore, F., Lassonde, M., Voss, P., Zatorre, R. J., and Belin, P. (2004). Pitch discrimination in the early blind. Nature 430, 309.

Gougoux, F., Zatorre, R. J., Lassonde, M., Voss, P., and Lepore, F. (2005). A functional neuroimaging study of sound localization: visual cortex activity predicts performance in early-blind individuals. PLoS Biol. 3, 324-333. doi: 10.1371/journal.pbio.0030027
Johnson, J. A., and Zatorre, R. J. (2005). Attention to simultaneous unrelated auditory and visual events: behavioural and neural correlates. Cereb. Cortex 15, 1609-1620.

Jones, B. (1975). Spatial perception in the blind. Br. J. Psychol. 66, 461-472.

Kawashima, R., O'Sullivan, B. T., and Roland,P.E. (1995). Positron-emission tomography studies of cross-modality inhibition in selective attention tasks: closing the "mind's eye". Proc. Natl. Acad. Sci. U.S.A. 92, 5969-5972.

Laurienti, P. J., Burdette, J. H., Wallace, M. T., Yen, Y. F., Field, A. S., and Stein, B. E. (2002). Deactivation of sensoryspecific cortex by cross-modal stimuli. J. Cogn. Neurosci. 14, 420-429.

Lessard, N., Paré, M., Lepore, F., and Lassonde, M. (1998). Early-blind human subjects localize sound sources better than sighted subjects. Nature $395,278-280$.

Lewald, J. (2002). Opposing effects of head position on sound localization in blind and sighted human subjects. Eur. J. Neurosci. 15, 1219-1224.

Lewald, J., Riederer, K. A., Lentz, T., and Meister, I. G. (2008). Processing of sound location in human cortex. Eur. J. Neurosci. 27, 1261-1270.

Merabet, L. B., Hamilton, R., Schlaug, G., Swisher, J. D., Kiriakopoulos, E. T., Pitskel, N. B., Kauffman, T., and Pascual-Leone, A. (2008). Rapid and reversible recruitment of early visual cortex for touch. PLoS ONE 3, e3046. doi: 10.1371/journal.pone.0003046

Merabet, L. B., and Pascual-Leone, A. (2010). Neural reorganization following sensory loss: the opportunity of change. Nat. Rev. Neurosci. 11, 44-52. Merabet, L. B., Swisher, J. D., McMains, S. A., Halko, M. A., Amedi, A., PascualLeone, A., and Somers, D. C. (2007). Combined activation and deactivation of visual cortex during tactile sensory processing. J. Neurophysiol. 97, 1633-1641.

Middlebrooks, J. C., and Green, D. M. (1991). Sound localization by human listeners. Annu. Rev. Psychol. 42, 135-159.

Morgan, M. (1999). Sensory perception: supernormal hearing in the blind? Curr. Biol. 9, R53-R54.

Pascual-Leone, A., and Hamilton, R. (2001). The metamodal organization of the brain. Prog. Brain Res. 134, 427-445.

Paus, T., Perry, D., Zatorre, R. J., Worsley, K., and Evans, A. (1996). Modulation of cerebral blood-flow in the human auditory cortex during speech: role of motor-to-sensory discharges. Eur. J. Neurosci. 8, 2236-2246.

Poirier, C., Collignon, O., De Volder, A. G., Renier, L., Vanlierde, A., Tranduy,
D., and Scheriber, C. (2005). Specific activation of $\mathrm{V} 5$ brain area by auditory motion processing: an fMRI study. Brain Res. Cogn. Brain Res. 25, 650-658.

Raichle, M., Martin, W., Herscovitch, P. Mintum, M., and Markham, J. (1983). Brain blood flow measured with intravenous $\mathrm{O}^{15} \mathrm{H}_{2} \mathrm{O}$. 1. Theory and error analysis. J. Nucl. Med. 24, 790-798.

Renier, L. A., Anurova, I., De Volder, A. G., Carlson, S., VanMeter, J., and Rauschecker, J. P. (2010). Preserved functional specialization for spatial processing in the middle occipital gyrus of the early blind. Neuron 68 138-148.

Röder, B., Teder-Sälejärvi, W., Sterr, A., Rösler, F., Hillyard, S. A., and Neville, H.J. (1999). Improved auditory spatial tuning in blind humans. Nature 400 , 162-166.

Shulman, G. L., Corbetta, M., Buckner R. L., Raichle, M. E., Fiez, J. A. L., and Petersen, S.E. (1997). Top-down modulation of early sensory cortex. Cereb Cortex 7, 193-206.

Silver, M. A., and Kastner, S. (2009) Topographic maps in human frontal and parietal cortex. Trends Cogn. Sci. 13, 488-495.

Szczepanski, S. M., Konen, C. S., and Kastner, S. (2010). Mechanisms of spatial attention control in frontal and parietal cortex. J. Neurosci. 30 148-160.

Talairach, J., and Tournoux, P. (1988). Co-planar stereotaxic atlas of the human brain: 3-dimensional proportional system. An approach to cerebral imaging. Stuttgart: Thieme.

Van Wanrooij, M. M., and Van Opstal,A. J. (2004). Contribution of head shadow and pinna cues to chronic monaural sound localization. J. Neurosci. 24, 4163-4171.

Voss, P., Collignon, O., Lassonde, M., and Lepore F. (2010). Adaptation to sensory loss. Wiley Interdiscip. Rev. Cogn Sci. 1, 308-328.

Voss, P., Gougoux, F., Lassonde, M., Fortin M., Guillemot, J. P., and Lepore, F. (2004). Early- and late-onset blind individuals show supra-normal auditory abilities in far space. Curr. Biol. 14, 1734-1738.

Voss, P., Gougoux, F., Lassonde, M. Zatorre, R. J., and Lepore, F. (2006). A PET study during auditory localization by late-onset blind individuals Neuroreport 17, 383-388.

Voss, P., Gougoux, F., Zatorre, R. J., Lassonde, M., and Lepore, F. (2008) Diffenrential occipital responses in early and late blind individuals during a sound-source discrimination task. Neuroimage 40, 746-758.

Wan, C. Y., Wood, A. G., Reutens, D. C. and Wilson, S. J. (2010). Early but not late-blindness leads to enhanced auditory perception. Neuropsychologia 48 , 344-348.

Weeks, R., Horwitz, B., Aziz-Sultan, A., Tian, B., Wessinger, C. M., Cohen, L. G., Hallett, M., and Rauschecker, J. P. (2000). A positron emission tomographic study of auditory localization in the congenitally blind. J. Neurosci. 20, 2664-2672.

Weisse, V., Stilla, R., Peltier, S., Hu, X., and Sathian, K. (2005). Shorterm visual deprivation alters neural processing of tactile form. Exp. Brain Res. 166 572-582.

World Health Organization. (1989). Tenth Revision of the International Classification of Disease, Vol. 2. Geneva: World Health Organization, 141.

Worsley, K., Evans, A., Marret, S., and Neelin, P. (1992). A three-dimensional statistical analysis for $\mathrm{CBF}$ activation studies in human brain. J. Cereb. Blood Flow Metab. 12, 900-918.

Zangenehpour, S., and Zatorre, R. J. (2010). Crossmodal recruitment of primary visual cortex following brief exposure to bimodal audiovisual stimuli. Neuropsychologia 48, 591-600.

Zatorre, R. J., Evans, A. C., and Meyer, E. (1994). Neural mechanisms underlying melodic perception and memory for pitch. J. Neurosci. 14, 1908-1919.

Zatorre, R. J., Mondor, T.A., and Evans, A. C. (1999). Auditory attention to space and frequency activates similar cerebral systems. Neuroimage 10, 544-554.

Zwiers, M. P., Van Opstal, A. J., and Cruysberg, J. R. M. (2001). A spatial hearing deficit in early blind individuals. J. Neurosci. 21, RC142-RC147.

Conflict of Interest Statement: The authors declare that the research was conducted in the absence of any commercial or financial relationships that could be construed as a potential conflict of interest.

Received: 13 December 2010; paperpending published: 07 February 2011; accepted: 14 March 2011; published online: 28 March 2011.

Citation: Voss P, Lepore F, Gougoux F and Zatorre RJ (2011) Relevance of spectral cues for auditory spatial processing in the occipital cortex of the blind. Front. Psychology 2:48. doi: 10.3389/fpsyg.2011.00048 This article was submitted to Frontiers in Auditory Cognitive Neuroscience, a specialty of Frontiers in Psychology.

Copyright (c) 2011 Voss, Lepore, Gougoux and Zatorre. This is an open-access article subject to an exclusive license agreement between the authors and Frontiers Media $S A$, which permits unrestricted use, distribution, and reproduction in any medium provided the original authors and source are credited. 